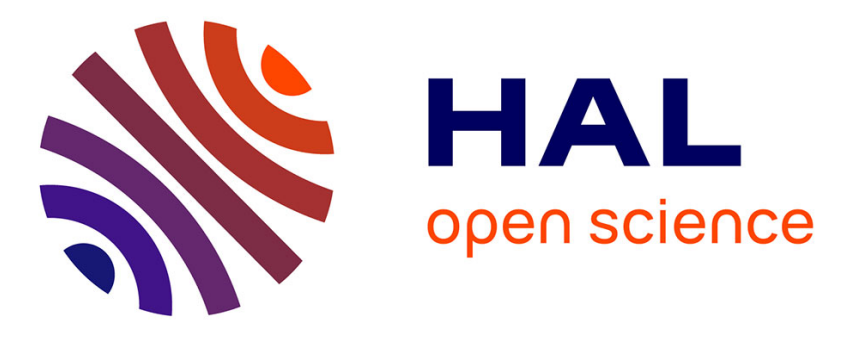

\title{
Ab initio calculations of the B1-B2 phase transition in $\mathrm{MgO}$
}

\author{
J. Bouchet, F. Bottin, V. Recoules, F. Remus, G. Morard, R. M Bolis, A. \\ Benuzzi-Mounaix
}

\section{- To cite this version:}

J. Bouchet, F. Bottin, V. Recoules, F. Remus, G. Morard, et al.. Ab initio calculations of the B1-B2 phase transition in MgO. Physical Review B: Condensed Matter and Materials Physics (1998-2015), 2019, 99 (9), pp.094113. 10.1103/PhysRevB.99.094113 . hal-02181694

\section{HAL Id: hal-02181694 \\ https://hal.sorbonne-universite.fr/hal-02181694}

Submitted on 12 Jul 2019

HAL is a multi-disciplinary open access archive for the deposit and dissemination of scientific research documents, whether they are published or not. The documents may come from teaching and research institutions in France or abroad, or from public or private research centers.
L'archive ouverte pluridisciplinaire HAL, est destinée au dépôt et à la diffusion de documents scientifiques de niveau recherche, publiés ou non, émanant des établissements d'enseignement et de recherche français ou étrangers, des laboratoires publics ou privés. 


\title{
Ab initio calculations of the B1-B2 phase transition in $\mathrm{MgO}$
}

\author{
J. Bouchet,${ }^{1}$ F. Bottin, ${ }^{1}$ V. Recoules,${ }^{1}$ F. Remus, ${ }^{1}$ G. Morard,${ }^{2}$ R.M. Bolis,${ }^{3,4}$ and A. Benuzzi-Mounaix ${ }^{3,4}$ \\ ${ }^{1}$ CEA, DAM, DIF, F-91297 Arpajon, France \\ ${ }^{2}$ Sorbonne Université, Institut de Minéralogie, de Physique des Matériaux et de Cosmochimie, \\ IMPMC, Museum National d'Histoire Naturelle, UMR CNRS 7590, IRD, Paris, France \\ ${ }^{3}$ LULI - CNRS, Ecole Polytechnique, CEA, Universit Paris-Saclay, Palaiseau cedex, F-91128, France \\ ${ }^{4}$ Sorbonne Université, UPMC Univ Paris 06, CNRS, \\ Laboratoire d'utilisation des lasers intenses (LULI), place Jussieu, Paris cedex 05, 75252, France
}

\begin{abstract}
We present an $a b$ initio study of $\mathrm{MgO}$ at high temperature and pressure, around the phase transition between the $\mathrm{B} 1$ and $\mathrm{B} 2$ phases. By means of ab initio molecular dynamic calculations, the thermal evolution of vibrational properties and thermodynamic quantities are obtained. We carefully compare our results with previous theoretical works on the phase transition curve and we analyse the differences among them. We show that anharmonic effects have been underestimated in the quasiharmonic approximation and that their inclusion in the free energy strongly straighten up the transition curve. Then we use our B1-B2 phase boundary and our calculated Hugoniot to analyze recent decaying shock experiments on $\mathrm{MgO}$. We also provide important thermodynamic quantities as the Grüneisen parameter and sound velocities and we discussed their temperature dependence.
\end{abstract}

\section{INTRODUCTION}

As one of the major component of the Earth's lower mantle, $\mathrm{MgO}$ has been extensively studied experimentally and theoretically. Since the $\mathrm{NaCl}$ (B1) structure is stable up to several megabars and thousand degrees Kelvin, $\mathrm{MgO}$ is also considered as an internal pressure standard and considerable efforts have been made to build its $P-V-T$ equation of state (EOS). With the discovery of super-Earths it is also crucial to extend the thermodynamic data of $\mathrm{MgO}$ up to pressures and temperatures expected in these massive exoplanets ${ }^{1}$, but also for the rocky core of gas and ice giants ${ }^{2,3}$. It has been theoretically predicted for a long time that $\mathrm{MgO}$ undergoes a phase transition between the rocksalt structure to the caesium chloride (B2) structure in the 400-600 GPa range ${ }^{4-12}$. Experimentally the transition has only been directly observed recently by dynamic X-ray diffraction measurements ${ }^{13}$, and its probable signature in laserdriven decaying ${ }^{14,15}$ and steady shocks experiments ${ }^{12,16}$ has been evidenced.

At $0 \mathrm{~K}$, the more recent $a b$ initio calculations are in close agreement and therefore they have narrower the range of the transition pressure between 475-510 $\mathrm{GPa}^{9-12}$, see Fig. 1. The small differences in the results can be incriminated to the flavor of the functional used to describe the exchange correlation term : $\mathrm{LDA}^{7,9,17}$ or GGA $\left(\mathrm{PBE}^{10,18}, \mathrm{AM} 05^{11,12,19}\right)$. At high temperature, the disagreement on the location of the phase boundary dramatically increases, from $7 \%$ at $0 \mathrm{~K}$, up to $40 \%$ at 8000 $\mathrm{K}$ between the lowest transition pressure $\left(\sim 310 \mathrm{GPa}^{12}\right)$ and the highest one $\left(\sim 440 \mathrm{GPa}^{10}\right)$, see Fig. 1. One of the goal of this paper is to understand these discrepancies and to propose a well-established phase transition boundary.

All the methods used to compute the phase transition line between the B1 and B2 structures are based on a decomposition of the Helmholtz free energy between the

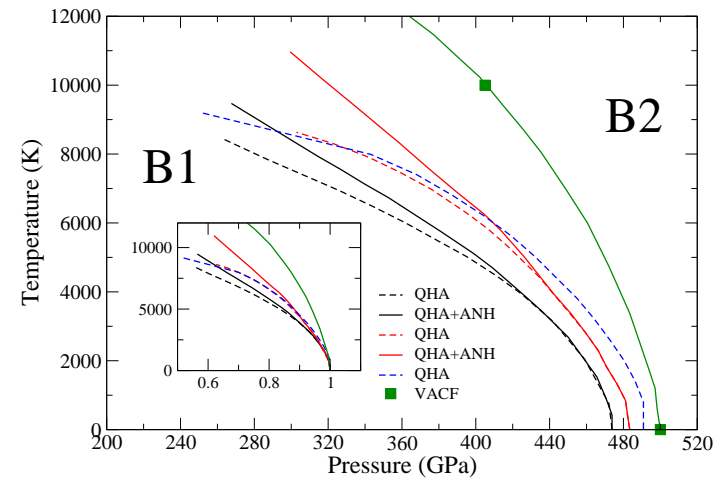

FIG. 1. (Color online) Theoretical B1-B2 phase boundary of $\mathrm{MgO}$. Dotted lines : quasiharmonic approximation of $\mathrm{S}$. Root et al. ${ }^{12}$ (black), A. B. Belonoshko et $a .^{9}{ }^{9}$ (blue) and D. Cebulla et $a .^{11}$ (red). The black and red lines include anharmonic corrections beyond the quasiharmonic approximation. The green squares are the results of $\mathrm{B}$. Boates et al. ${ }^{10}$ using velocity autocorrelation function and the green line a Kechin fit to these data. The inset shows the renormalisation of the thermal transition pressures by the athermal one.

static part, or cold curve, obtained at $0 \mathrm{~K}$ and the ionic or vibrational contribution coming from the atomic motions in temperature. Since the methods are in good agreement for the transition pressure at $0 \mathrm{~K}$, the differences are due to the way of obtaining the vibrational part. The key ingredient of this contribution is the phonon spectra, or rather the number of phonon states by energy, the phonon density of states (PDOS) that can be used to calculate the vibrational free energy. The phonon spectra can be obtained at $0 \mathrm{~K}$ using either the density functional perturbation theory ${ }^{20-22}$ (DFPT) or the finite displacements method ${ }^{23}$. Based on these calculations 
performed at several volumes, the so called quasiharmonic approximation $^{24}$ (QHA) introduces a temperature dependence of the phonon frequencies through thermal expansion that alters the volume of the solids. Applied to $\mathrm{MgO}^{6,9}$, the QHA predicts the lowest phase boundary and favors the B2 phase over the B1 phase at high temperature with a close to zero Clausius-Clapeyron slope above $8000 \mathrm{~K}$, see Fig. 1. Despite its usefulness and wide application, the QHA suffers of two major flaws: (i) it cannot be applied if the crystal structure is dynamically unstable at $0 \mathrm{~K}$, as the bcc phase of $\mathrm{Zr}^{25}, \mathrm{Li}^{26}$, or the actinides ${ }^{27,28}$ and (ii) it neglects the intrinsic thermal effects in opposition to the extrinsic volume dependence.

To test the robustness of the QHA, A. Glensk et al. ${ }^{29}$ have recently studied the anharmonicity of several fcc metals using upsampled thermodynamic integration ${ }^{30}$. They show the importance of the anharmonic effects, even at temperatures far below the melting point. More specifically, the validity domain of the QHA for $\mathrm{MgO}$ has been studied by A. Erba et al. ${ }^{31}$ and Z. Wuet al..$^{32}$ by a carefull comparison with experimental data for several thermodynamic quantities. At room pressure, they estimate that the QHA is valid up to about $1000 \mathrm{~K}$, and up to $3000 \mathrm{~K}$ at $100 \mathrm{GPa}$ (around $30-40 \%$ of the melting temperature). This domain of validity of the QHA has to be taken cautiously, particularly when looking at phase transitions where differences of a few $\mathrm{meV}$ in the Gibbs free energy can induce a difference of several GPa on the transition pressure. Basically the QHA will fail if the phonon frequencies have a strong temperature dependence, i.e when atomic vibrations increase in magnitude compared to the atomic distance, as for example close to the melting point but also at a phase transition where soft modes can appear.

To correct the QHA, D. Cebulla et al. ${ }^{11}$ and S. Root et $a l .{ }^{12}$ have used $a b$ initio molecular dynamics (AIMD) but their methods still relies on the QHA to deal with temperatures where anharmonicity has to be included in the estimation of the free energy. Nonetheless, their results show that the QHA overestimates the stability domain of the B2 phase in temperature and that the anharmonic effects drive to higher transition pressures at higher temperatures, see Fig. 1. Using Fourier analyzing of the velocity autocorrelation function from AIMD B. Boates et al. ${ }^{10}$ observe a similar effect but with a stronger intensity at $10000 \mathrm{~K}$. Although this method should in principles include the whole anharmonicity present in the AIMD it requires long simulations and large supercells to avoid finite-size effects, as when employed with classical molecular dynamics. In Ref. [10], they used supercells of 64 and 128 atoms to simulate the B1 and B2 phases, rather small numbers compared to the several thousand atoms used in classical MD.

In the past ten years, strong efforts have been made to take into account explicit temperature effects and major advances have been obtained. New methods capturing the thermal properties of solids at non-zero temperature are now available and can be applied with $a b$ initio calculations. These approaches combine ideas including finite large displacements, molecular dynamics sampling, self consistent harmonic theories, and different force fitting schemes. The most widely used methods include Self-Consistent Ab Initio Lattice Dynamics $(\mathrm{SCAILD})^{25}$, Stochastic Self-Consistent Harmonic Approximation (SSCHA) $)^{33,34}$, Temperature Dependent Effective Potential (TDEP) ${ }^{26,35,36}$, Anharmonic LAttice MODEl (ALAMODE) ${ }^{37}$, Compressive Sensing Lattice Dynamics $^{38}$. Other methods obtain anharmonic contributions via a derivation of the Gibbs energy ${ }^{29}$, or a series expansion of the interatomic forces constants ${ }^{39}$. A large number of new phenomena, intrinsically temperature dependent, can now be captured: the modification of the PDOS and free energy, the (T,P) phase transition boundaries, the evolution of elastic constants or Grüneisen coefficients, the phonon lifetimes, the thermal conductivity...

Here we have used the TDEP technique developed by O. Hellman and coworkers ${ }^{26,35,40}$ to extract the vibrational frequencies from AIMD simulations, with an original implementation in the ABINIT package ${ }^{41,42}$. This method takes roots in the pionner work of Hooton ${ }^{43}$ and K. Esfarjani et al. ${ }^{39}$ and has already been successful to describe phase transitions at high temperature as for $\mathrm{Ti}$ and $\mathrm{Zr}^{26,44},{ }^{4} \mathrm{He}^{35}$ or $\mathrm{U}$ and $\mathrm{Pu}^{27,28}$. In TDEP, a Taylor expansion on the atomic displacements is used to fit the Born-Oppenheimer molecular dynamics potential energy surface at finite temperature and to obtain the interatomic force constants (IFC). The second order IFCs give the phonon frequencies while the higher order terms can be used to calculate phonon lifetimes, thermal conductivity or the Grüneisen parameter.

The paper is structured as follows. In Sec. II we give the details of the AIMD simulations and the construction of the free energy. In Sec. III we give our results for the B1-B2 transition curve and we compare our results with previous calculations and recent experiments. This is followed by the presentation of several thermodynamics quantitites including thermoelasticity, Grüneisen parameter and wave velocities.

\section{SIMULATION DETAILS}

Simulations were performed using the ABINIT package $^{41,42}$ in the framework of the Projector Augmented Wave (PAW) method ${ }^{45,46}$ and by means of the local density approximation (LDA ${ }^{17}$. Using ATOM$\mathrm{PAW}^{47-49}$, we generated a PAW atomic data with a radius $r_{\text {PAW }}$ equals to $1.0 \AA$, with $2 s, 2 p, 3 s$, and $3 p$ states as valence electrons (10 electrons) for $\mathrm{Mg}$ and a radius $r_{\text {PAW }}$ equals to $0.6 \stackrel{A}{A}$, with $2 s$ and $2 p$ states as valence electrons (6 electrons) for O. The cutoff energy chosen for the plane wave set along the simulations is $816 \mathrm{eV}$. To determine the B1-B2 phase boundary we need to calculate the free enthalpy $G$ of both phases, with

$$
G(T, P)=F(T, P)+P V(T, P),
$$


where the free energy $F$ is given by

$$
F(T, V)=E_{0}(V)+F_{\mathrm{vib}}(V, T)+F_{\mathrm{el}}(V, T) .
$$

Here $E_{0}(V)$ is the energy of the static lattice, $F_{\text {vib }}$ the vibrational free energy due to the atomic motions and $F_{\text {el }}$ the electronic free energy. For the static calculations, at $\mathrm{T}=0 \mathrm{~K}$, we use the unit cells of the $\mathrm{B} 1$ and the $\mathrm{B} 2$ phases with two atoms and a $8 \times 8 \times 8$ Monkhorst-Pack mesh.

To obtain the vibrational part we use two methods, the QHA and the TDEP. They differ in the way of calculating the interatomic force constants (IFCs). The QHA is based on static calculations, so at $0 \mathrm{~K}$, involving only the unit cells and the DFPT. The dynamical matrices are calculated on a grid of $\mathbf{q}$ points and then Fourier transformed, yielding to the IFCs. With this method, the phonon frequencies are only dependent of the volume. Our calculations were performed on $4 \times 4 \times 4$ grid of $\mathbf{q}$ points in the Brillouin zone. The second method, the TDEP $^{26,35}$, calculates directly the IFC in real space from sets of forces and displacements obtained from AIMD. For this purpose, we use supercells of 128 atoms for the B1 and B2 phases, corresponding to $4 \times 4 \times 4$ rhombohedral B1 unit cells and cubic B2 unit cells (we also use larger and smaller supercells for convergence studies, see below Fig. 4). Note that contrary to previous calculations $^{10-12}$, also based on AIMD, we use the same number of atoms for the two structures. Our AIMD were performed with a $2 \times 2 \times 2$ Monkhorst-Pack mesh leading to the inclusion of 4 special $\mathbf{k}$-points. This mesh was checked against a $3 \times 3 \times 3$ Monkhorst-Pack mesh to ensure the required accuracy, less than $1 \mathrm{meV}$ on the vibrational free energy. Simulations were performed in the $N V T$ ensemble (constant number of particles, constant volume and temperature) and were run for about $5 \mathrm{ps}$ using a time step $(\tau)$ of $0.60 \mathrm{fs}$. Taking into account the whole symmetries of the system, we have to calculate 19 coefficients for the B1 phase (up to the 8 th shell of nearest neighbours) and 21 coefficients for the B2 phase (up to the 9th shell of nearest neighbours) to build the IFC matrices with the TDEP. So, contrary to the DFPT which uses unit cells, the TDEP requires AIMD on supercells of atoms to obtain the IFCs at a sufficiently large distance where they become negligible. It is therefore much more expensive in terms of computational time but goes beyond the QHA since the phonon frequencies are not only volume dependent but also explicitly temperature dependent.

Once the IFC are obtained, by either methods, a Fourier transform is performed to get the dynamical matrix at any q-point of the Brillouin zone and to calculate the PDOS. Then the free energy is given by :

$F_{\mathrm{vib}}(V, T)=k_{B} T \int_{0}^{\infty} d \omega g(\omega(V, T)) \ln \left[2 \sinh \left(\hbar \omega(V, T) / 2 k_{B} T\right.\right.$

where the temperature dependence of the phonon frequencies, $\omega$, and the PDOS, $g(\omega)$, is only valid for the

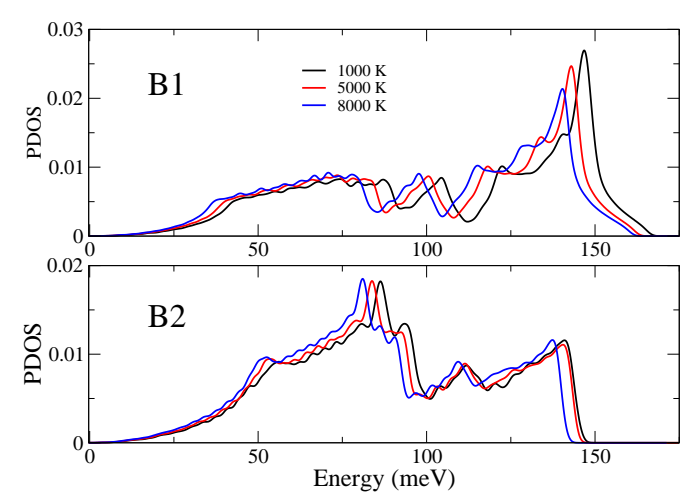

FIG. 2. (Color online) PDOS of the B1 and B2 phases of $\mathrm{MgO}$ at $6.75 \mathrm{~g} / \mathrm{cm}^{3}$ and for temperatures of 1000,5000 and $8000 \mathrm{~K}$.

TDEP method. Once the free energies are calculated with Eq. 2, they are fitted by a Vinet EOS to obtain their pressure dependence and then the free enthalpy with Eq. 1.

\section{B1-B2 PHASE TRANSITION}

\section{A. QHA versus TDEP method}

We show in Fig. 2 the variation of the PDOS as a function of temperature obtained with TDEP for the $\mathrm{B} 1$ and $\mathrm{B} 2$ phases at a fixed density of $6.75 \mathrm{~g} / \mathrm{cm}^{3}$. For both structures, the whole spectra softens with temperature. This softening is a signature of explicit thermal effects, and since in QHA the spectrum is independent of the temperature at fixed volume, it will induce lower vibrational free energies in TDEP. This is directly seen in Fig. 3 where we compare the free energies obtained with both methods. Up to about $2000 \mathrm{~K}$, both methods give the same free energies, but at higher temperatures the free energies obtained with TDEP deviate from the QHA. More importantly, the free energies difference, $\Delta F_{\mathrm{vib}}=F_{\mathrm{vib}}^{\mathrm{B} 1}-F_{\mathrm{vib}}^{\mathrm{B} 2}$ is smaller in TDEP, as shown in the inset of Fig. 3. This means that the QHA overestimates the stability domain of the B2 phase compared to the TDEP method. In other words, the intrinsic anharmonic effects will move the phase boundary at higher pressure at high temperature, in agreement with previous works $^{10-12}$.

We have also applied the TDEP method on several supercell sizes: 64, 128 and 216 atoms for the B1 phase and 54, 128 and 250 atoms for the B2 phase. The vibrational free energy differences between the B1 and B2 phases, $\Delta F_{\mathrm{vib}}$, are shown on Fig. 4 as a function of the supercell sizes. At $2000 \mathrm{~K}$, the effect of the supercell size is negligible, and the TDEP method is close to the QHA 


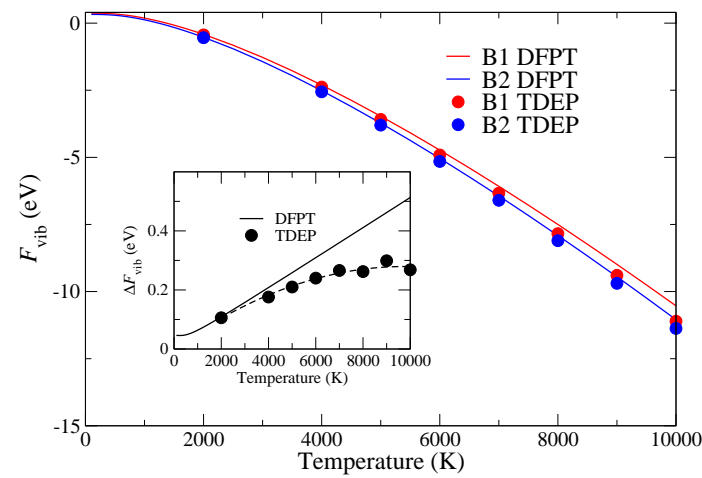

FIG. 3. (Color online) Comparison of the vibrational free energies of the $\mathrm{B} 1$ (in red) and $\mathrm{B} 2$ (in blue) phases of $\mathrm{MgO}$ obtained with DFPT (lines) and TDEP (filled circles) at $7.75 \mathrm{~g} / \mathrm{cm}^{3}$. Inset : difference between the free energies of the B1 and the B2 phases in QHA (solid line) and with the TDEP method (filled circles and dotted line)

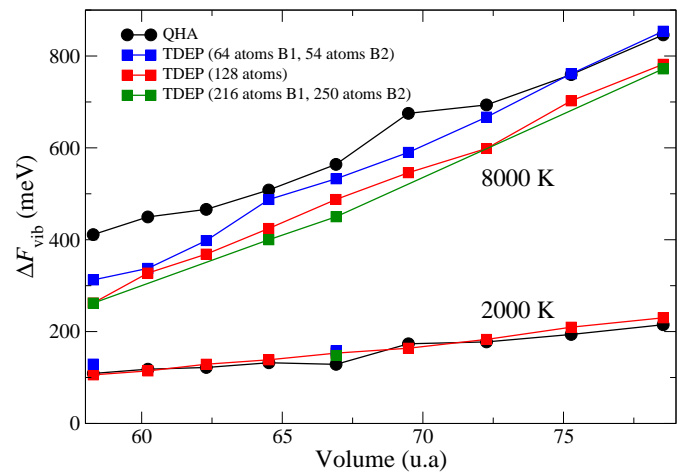

FIG. 4. (Color online) vibrational free energy differences between the B1 and B2 phases at 2000 and $8000 \mathrm{~K}$ for the QHA (black circles) and with the TDEP method as a function of the supercells size.

for all the volumes considered. At $8000 \mathrm{~K}$ we observe a size effect. The smaller supercell sizes overestimate the free energy differences, favoring the B2 phase, while the calculations with 128 atoms and more are in agreement. We also observe a larger difference between TDEP and QHA for smaller volumes (larger pressure). This seems in contradiction with the belief that anharmonic effects should decrease with pressure along an isotherm. In fact, it is not. For both phases, the difference between QHA and TDEP decreases with the volume, but not in the same ratio : $F_{\text {vib }}$ of the $\mathrm{B} 2$ phase becomes closer to the QHA value than for the B1 phase.

Our results for the phase transition boundary are presented in Fig. 5 in comparison with the previous the-

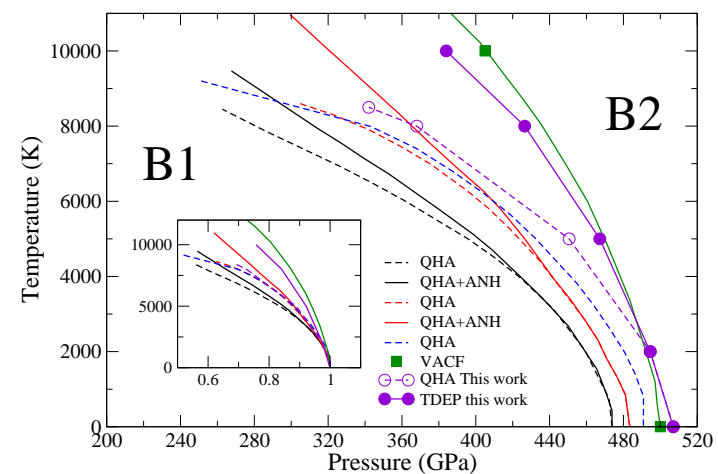

FIG. 5. (Color online) Same as Fig. 1, with our QHA results (purple open circles and dotted line) and TDEP results (purple filled circles and solid line).

oretical data of Fig. 1. First, our QHA results are in agreement with the work of A. B. Belonoshko et al. ${ }^{9}$ and D. Cebulla et al. ${ }^{11}$ with similar slopes of phase boundary (see the inset of Fig. 5). We therefore observe the same discrepancy with the QHA data of S. Root et al. ${ }^{12}$. The phase boundary predicted with the TDEP method is steeper, increasing the stability domain of the B1 phase at high temperature. Consequently, our curve is in agreement with the one obtained by B. Boates et al. ${ }^{10}$ using the VACF method and which, in principle, should take into account the full anharmonicity of the simulated system. The previous attempts to go beyond the $\mathrm{QHA}^{11,12}$ and to include the intrinsic anharmonicity, predicted also an extension of the stability domain of the B1 phase, but to a lesser extent than the one that we obtain with the TDEP. In the next session we analyze these discrepancies and we will try to reconcile, if possible, the different methods.

\section{B. Comparison with previous calculations}

D. Cebulla et al. ${ }^{11}$, propose to take into account the anharmonicity by expressing the internal anharmonic energy $U_{\text {anh }}$ with :

$$
U_{\mathrm{anh}}=U_{\mathrm{AIMD}}+E_{\mathrm{KIN}}-U_{\mathrm{vib}}-E_{0}
$$

where $U_{\mathrm{AIMD}}$ and $E_{\mathrm{KIN}}$ are respectively the internal and kinetic energy of the AIMD, $E_{0}$ the electronic ground state energy and $U_{\text {vib }}$ is the harmonic internal energy given by :

$$
U_{\mathrm{vib}}(V, T)=\int_{0}^{\infty} d \omega g(\omega(V, T)) \hbar \omega(T) / 2 \operatorname{coth}\left(\hbar \omega(V, T) / 2 k_{B} T\right) .
$$




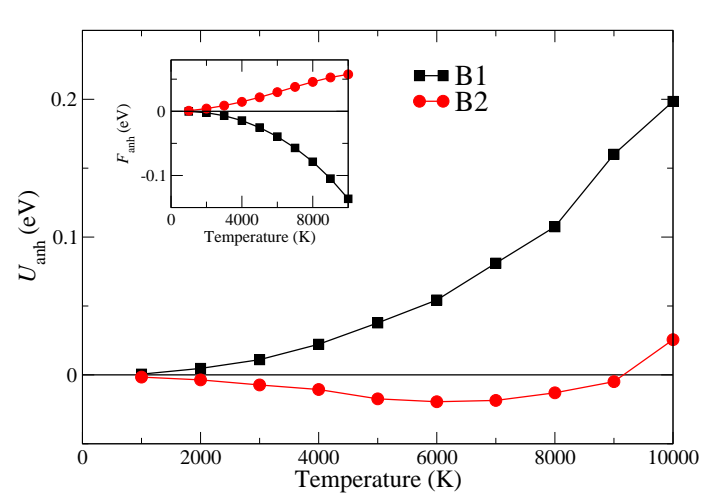

FIG. 6. (Color online) Internal anharmonic energy $U_{\text {anh }}$ for the B1 (in black) and B2 (in red) phases as a function of temperature obtained with Eq. 7 at $6.75 \mathrm{~g} / \mathrm{cm}^{3}$. Inset : $F_{\text {anh }}$ as a function of temperature.

Then the anharmonic free energy at a temperature $T_{f}$ is obtained via a thermodynamic integration from an initial temperature $T_{i}$ :

$$
F_{\mathrm{anh}}\left(T_{f}\right)=F_{\mathrm{anh}}\left(T_{i}\right) \frac{T_{f}}{T_{i}}-T_{f} \int_{T_{i}}^{T_{f}} \frac{U_{\mathrm{anh}}(T)}{T^{2}} d T
$$

Since $F_{\text {anh }}\left(T_{i}\right)$ is not known, the idea is to infer a temperature where this energy is negligible and to eliminate the first term of the right hand side of Eq. 6. This temperature has also to be high enough to neglect the zero point motion energy contained in $U_{\text {vib }}$ but not taken into account in $U_{\mathrm{AIMD}}$ since the atomic motions follow a classical trajectory. In the same way, the temperature should be high enough so that the kinetic part contained in $U_{\mathrm{vib}}$ reaches the classical value of $3 k_{B} T$ given by the AIMD. D. Cebulla et al. ${ }^{11}$ chose a value of $T_{i}=3000 \mathrm{~K}$. At this temperature and above, since the QHA and the TDEP are based on the harmonic approximation, $U_{\text {vib }}$ will be equal in both models to the classical value of $3 k_{B} T$, and obviously, Eq. 6 will give the same correction. In other terms, this method is completely independent of the shape of the PDOS (see Fig. 2). So it clearly neglects the fact that the phonon frequencies are temperature dependent and it will not correct $F_{\text {vib }}$ : this intrinsic anharmonicity will still be missing in $F_{\text {anh }}$. Therefore, the QHA and the TDEP cannot be reconciled with this method. But it can be used to estimate the anharmonic part not included in the TDEP.

To avoid the problem of the zero point motion and the kinetic energy we can use the harmonic potential energy $^{35}$ directly calculated with the IFCs obtained with the TDEP to calculate the anharmonic internal energy :

$$
U_{\mathrm{anh}}=U_{\mathrm{AIMD}}-1 / 2 \sum_{i j} \Phi_{i j} u_{i} u_{j}-E_{0}
$$

where the $\Phi_{i j}$ are the IFCs and $u_{i}$ the atomic displacements. We present in Fig. 6 the internal anharmonic energy as a function of temperature and for a density of $6.75 \mathrm{~g} / \mathrm{cm}^{3}$. At low temperature $U_{\text {anh }}$ converges towards zero, the atomic motions in both phases are purely harmonic. As the temperature increases, anharmonic effects arise and strongly depend of the structure. For the B2 phase, $U_{\text {anh }}$ is negative and close to zero, while it strongly increases with temperature for the B1 phase. This shows again that anharmonic effects are more important in the $\mathrm{B} 1$ phase than in the $\mathrm{B} 2$ one and that even at high pressure, these effects cannot be neglected. Following Eq. 6, we can calculate $F_{\text {anh }}$, see the inset of Fig. 6. Compared to the QHA, the intrinsic anharmonic effects described by the TDEP lower the vibrational free energy difference between the B1 and B2 phases, see Fig. 3, pushing the phase boundary to higher pressure at high temperature. If we add $F_{\text {anh }}$ to $F_{\text {vib }}$ obtained with TDEP, it will have a cumulative effect and the phase boundary will steepen even further. For the B1 phase, $F_{\text {anh }}$ counts for about $20 \%$ of the anharmonic correction to the QHA, the rest being given by the TDEP, while for the B2 phase, $F_{\text {anh }}$ is positive, so slightly brings back the TDEP toward the QHA value by $5 \%$.

S. Root et al. ${ }^{12}$ propose an other approach to go beyond the QHA based on a thermodynamic integration (TI) to calculate the change in entropy along an isochore

$$
\Delta S=\int_{T_{i}}^{T f} \frac{1}{T}\left(\frac{\partial U_{\mathrm{AIMD}}}{\partial T}\right)_{V} d T
$$

The main drawback of this method is obviously the number of simulations required to obtain the entropy at high temperature. The authors used an increment of 250 $\mathrm{K}$, which means that to have the entropy at $8000 \mathrm{~K}$, starting from $1000 \mathrm{~K}$, about 30 AIMD simulations are necessary. Multiplied by the number of isochores, the computational cost can rapidly become too cumbersome.

We have compared this method with the TDEP one on several isochores, using the same supercell sizes as in Ref. 12, 64 and 54 atoms in the B1 and B2 phases respectively. An example of the entropy differences between the two phases is given in fig. 7 for $\rho=7.75 \mathrm{~g} / \mathrm{cm}^{3}$. In QHA this difference is a straight line, proportional to the temperature. Note that we have neglected the zero point motion and other quantum effects not included in eq. 8, but they have a small impact and only at low temperatures. The agreement between TDEP, eq. 8 and QHA is excellent up to $2000 \mathrm{~K}$ where we start to observe a deviation. As the temperature increases, the entropy difference between $\mathrm{B} 1$ and $\mathrm{B} 2$ diminishes compared to the QHA, as already shown in fig. 3. The effect is stronger with eq. 8 than with TDEP, meaning that it will push the phase boundary to higher pressures than the ones obtained with TDEP. A result in contradiction with the lower pressures reported by S. Root et al. ${ }^{12}$, see Fig. 5 . 


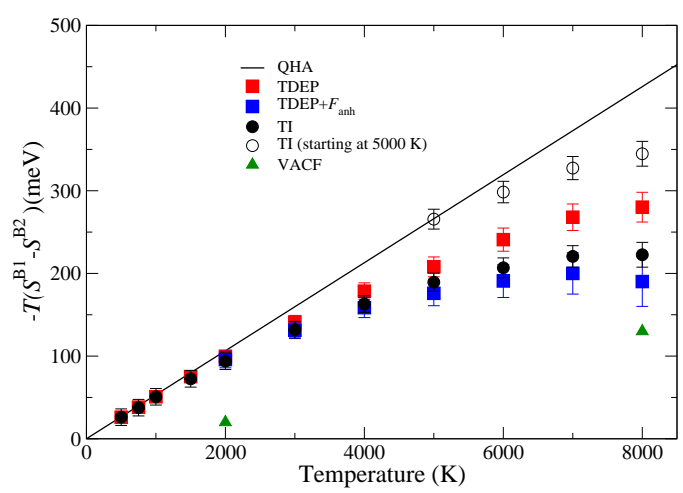

FIG. 7. (Color online) Entropy difference between the B1 and $\mathrm{B} 2$ phases at $7.75 \mathrm{~g} / \mathrm{cm}^{3}$ as a function of the temperature. The solid line corresponds to the QHA, the filled black circles to the TI of Eq. 8 and the red squares to TDEP. The blue squares are similar to the red ones with the correction $F_{\text {anh }}$ of Eq. 6 while the open black circles corresponds to an TI starting at $5000 \mathrm{~K}$. The green triangles are the results of the VACF at 2000 and $8000 \mathrm{~K}$. The error bars have been estimated with respect to calculation with supercell sizes of 216 atoms and 250 atoms for the B1 and the B2 structures respectively.

To understand these discrepancies, we have firstly checked the influence of the exchange correlation functional, since S. Root et al. ${ }^{12}$ use the Armiento-Mattson $(\text { AM05 })^{19}$ while we use the LDA. The vibrational free energies obtained with TDEP are slightly different (few meV), but in favor of the B1 phase with AM05 compared to LDA. Therefore, the discrepancies that we observe compared to Ref. 12 can not be attributed to the choice of the exchange correlation functional. S. Root et al. ${ }^{12}$ mentioned deviation with the QHA around $5000 \mathrm{~K}$ and $400 \mathrm{GPa}$, while we already observed an effect at 2000 $\mathrm{K}$. If we use this value of $5000 \mathrm{~K}$ as the starting temperature for the TI, we underestimate the entropy differences, as shown by the open circles in fig. 7. The consequence is of course a phase transition at pressures closer to the ones predicted by QHA and therefore by S. Root et al. ${ }^{12}$. We have also performed calculations with bigger supercells, 128 and 216 atoms for the B1 phase, 128 and 250 atoms for the B2 phase. At $2000 \mathrm{~K}$ the internal energy is converged to $1 \mathrm{meV}$ as a function of the supercell size but at $8000 \mathrm{~K}$ we observe differences up to $5 \mathrm{meV}$. This supercell size effect is similar to the one that we observe for the TDEP method, see Fig. 4, where we have shown that a small supercell favors the B2 phase.

Since, Eq. 8 is based on the internal energy coming from AIMD simulations with no further approximation as in TDEP, it should in principle contained the whole anharmonicity of the simulated system. The difference that we observe between TI and TDEP is close to $F_{\text {anh }}$ obtained with the method of D. Cebulla et al. ${ }^{11}$ and if we add this correction to the entropy found in TDEP we almost recover the TI, see Fig. 7. This confirms that the anharmonic terms that we do not take into account with TDEP correct to a larger extend the QHA.

B. Boates et $a l .{ }^{10}$ used the Fourier transforms of the velocity autocorrelation functions (VACF) to calculate the vibrational entropies. They obtained a transition pressure of $405 \mathrm{GPa}$ at $10000 \mathrm{~K}$, close to the value obtained with the TDEP method, see Fig. 5. This method is usually used with classical MD, since it requires large supercells and considerably long simulation time to obtain the PDOS. B. Boates et al. ${ }^{10}$ used 64 and 128 atoms only in their supercells for the B1 and B2 phases respectively. We have performed simulations up to $20 \mathrm{ps}$ for several $\rho, T$ points to obtain the VACF and to calculate the vibrational free energies. This lead us to think that the supercell sizes used in Ref. 10 are not large enough to converge the free energy. At $2000 \mathrm{~K}$ and $7.75 \mathrm{~g} / \mathrm{cm}^{3}$ for example, a temperature where anharmonicity should be negligible, we do not recover the QHA result, due to a strong underestimation of the vibrational free energy of the B1 phase (100 meV), partially compensated by a similar effect on the B2 phase $(40 \mathrm{meV})$, see Fig. 7. At $8000 \mathrm{~K}$, it is slightly better, but again, certainly due to a cancelation of errors between the two phases.

To sum up, we are now able to explain the discrepancies between our calculations and the previous ones. These differences come from the treatment of the intrinsic anharmonic part for D. Cebulla et al. ${ }^{11}$ or by an overestimation of the temperature at which the anharmonic effects have to be taken into account for S. Root et al. ${ }^{12}$. Concerning the work of B. Boates et al. ${ }^{10}$, we do not recover their results.

\section{Comparison with experiments}

Fig. 8 shows the experimental $P-T$ curves with our calculated B1-B2 transition line and Hugoniot. We plot also recent $a b$ initio predictions of the melting curve ${ }^{9,12}$. Recently, two decaying shock experiments ${ }^{14,15}$ have studied the phase diagram of $\mathrm{MgO}$. In the first one ${ }^{14}$, a clear bump and a slight slope change were observed in the $P-T$ curve along the Hugoniot. The strong bump at $450 \mathrm{GPa}$, was interpreted as the B1-B2 phase transition and the second one at $650 \mathrm{GPa}$, much softer, as the B2melt. The second experiment ${ }^{15}$, reported only the first bump, at similar thermodynamic conditions, but did not observe the slope change at higher pressure. Contrary to Ref. 14, they interpreted this feature as the B2-melt and not the B1-B2 solid phase transition, similarly to interpretation of decaying shock experiments performed on $\mathrm{SiO} 2^{50}$ or $\mathrm{MgSiO}^{51}$. In addition, with impact shock compression experiments, S. Root et al. ${ }^{12}$ observed only one breack in the Hugoniot around 360 GPa. Note that if a bump along the Hugoniot can be attributed to a phase transition the nature of this phase transition cannot be determined in the absence of microscopic in situ diagnos- 
tics. Can our calculations help to solve this controversy?

Our predicted B1-B2 phase boundary is close to the bump observe in the decaying shock experiments at 450 GPa. Knowing that anharmonic effects not included in our calculations should increase the stability domain of the B1 phase (see the discussion in the previous section), a nice correlation between the bump observed in decaying shock and the B1-B2 phase transition could be deduced. However, different arguments are on the side of a melting observation during decaying shock experiments. Firstly, the low intensity or absence of a second bump related to melting signature in $\mathrm{S}$. McWilliams et $a l^{14}$ is ambiguous, as a large thermodynamic domain is investigated (up to $1 \mathrm{TPa}$ and $30000 \mathrm{~K}$ ) far above the predicted melting line ${ }^{9,10,12,16,52}$. Secondly, there is a strong disagreement between the calculated Hugoniot for the B2 phase and the experimental points above $450 \mathrm{GPa}$, as already noted by D. Cebulla et al. ${ }^{11}$. The theoretical Hugoniot in the B1 domain is consistent with the lower part of the experimental results (below $450 \mathrm{GPa}$ ). After the bump, the experimental points are close to our calculated liquid Hugoniot. This comparison between our theoretical and experimental Hugoniots seems therefore to indicate a direct transition between the B1 and the liquid phase along the Hugoniot, close to the triple point, with a melting point around $450 \mathrm{GPa}$ and $10000 \mathrm{~K}$ as proposed in Ref[15]. This melting point is close to the computed melting line of the B1 phase of N. de Koker et $a l .{ }^{53}$, using a comparison of the Gibbs free energies of the solid and the liquid and based on AIMD, but also with extrapolation of the B1 melting line obtained with the $\mathrm{Z}$ method by A. B. Belonoshko et al. ${ }^{9,54}$ and with the two phase calculations of S. Root et al. ${ }^{12}$ and R. Musella et $a l .{ }^{55}$. But in this region, the calculated melting line of the $\mathrm{B} 2$ phase is above the results for the $\mathrm{B} 1$ phase $^{12}$, see Fig. 8.

Ab-initio calculations of melting curves are difficult and their uncertainties can be around several hundred kelvins. These uncertainties are cumulative when comparing melting lines obtained with different structures. In our simulations, at $6.0 \mathrm{~g} / \mathrm{cm}^{3}$ and $10000 \mathrm{~K}$, corresponding to a pressure of $330 \mathrm{GPa}$, the B2 structure melts spontaneously (see the red cross on Fig. 8), contrary to the B1 structure, while at the same density and $9000 \mathrm{~K}$ (283 GPa), the B2 structure stays solid. This melting of the perfect crystal along an isochore is known as the heat until it melts method and generally induces a superheating that results in an overestimation of the melting temperature ${ }^{56}$. New simulations, with larger supercells and with new methods to determine the melting are necessary to clarify this debate. In parallel, shock or diamond anvil cells experiments, coupled with X-ray diffraction, are strongly needed.

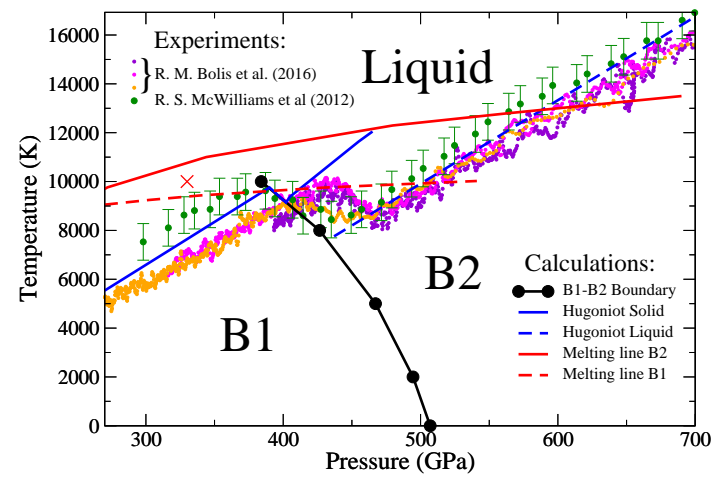

FIG. 8. (Color online) Experimental shock temperature data in comparison with our predicted B1-B2 phase boundary. Purple, magenta and orange dots : R. M. Bolis et al. ${ }^{15}$, green dots: R. S. McWilliams et al. ${ }^{14}$. The black solid line with filled circles is the B1-B2 boundary obtained with TDEP and the blue line the calculated Hugoniot. The dashed blue line is an extension of our Hugoniot curve for the liquid phase in the stability domain of the solid phase. The red curve is the calculated melt line of S. Root et al. ${ }^{12}$ for the B2 structures and the red dashed one is the calculated melt line of $\mathrm{N}$. de Koker et $a l .{ }^{53}$ for the B1 structure. The red cross indicates the melting of an AIMD with B2 as the starting structure of the simulation.

\section{THERMODYNAMICS PROPERTIES}

One of the main advantage of using the TDEP method is that we can not only calculate a temperature dependent free energy but also extract from the AIMD various thermodynamical quantities as the thermoelasticity, the Grüneisen parameters or the thermal expansion.

\section{A. Thermoelasticity}

The elastic constants can be obtained from the secondorder force constants following ${ }^{57-59}$ :

$$
C_{\alpha \beta \gamma \delta}=A_{\alpha \gamma \beta \delta}+A_{\beta \gamma \alpha \delta}-A_{\alpha \beta \gamma \delta}
$$

with,

$$
A_{\alpha \beta \gamma \delta}=\frac{1}{2 V} \sum_{i j} \Phi_{i j}^{\alpha \beta} d_{i j}^{\gamma} d_{i j}^{\delta}
$$

where $d_{i j}^{\alpha}$ is the $\alpha$ th component of the distance between the atom $i$ and $j$. The full elastic constants matrix, $C_{\alpha \beta \gamma \delta}$, can be reduced following the symmetries and the Voigt notation. The temperature effects are then directly introduced in the elastic constants by the temperature variation of the IFCs. As shown by N. Shulumba et al. ${ }^{59}$, this method is much faster and practical than the one using deformation matrix which needs several AIMD simulations or based on the QHA to calculate the free energies 


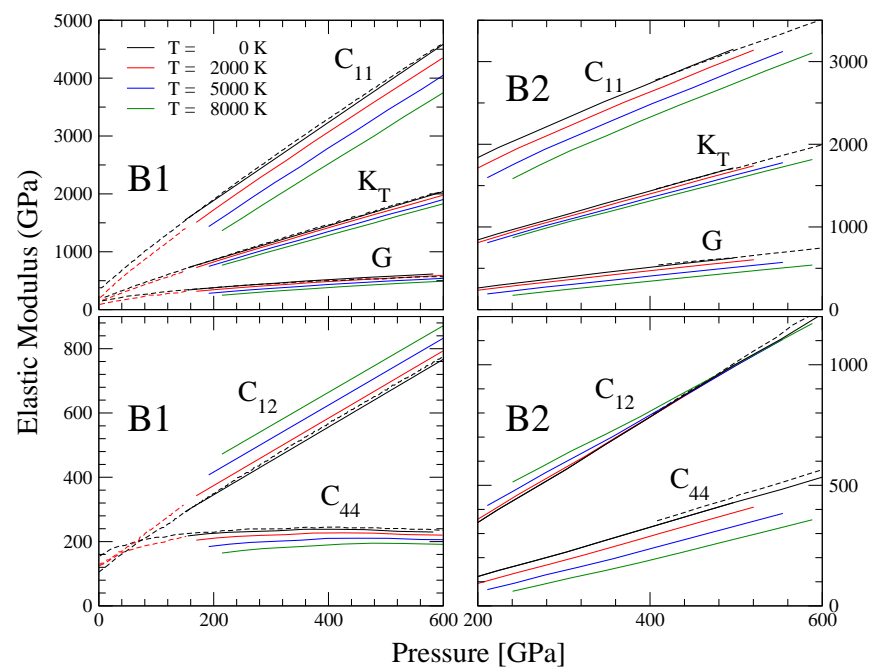

FIG. 9. (Color online) Elastic constants, $C_{11}, C_{12}$ and $C_{44}$, isotropic bulk $\left(K_{T}\right)$ and shear $(G)$ moduli of $\mathrm{MgO}$ of the B1 and B2 phases as a function of pressure and temperature. The dashed lines are the results of Karki et al. ${ }^{60,61}$ at $0 \mathrm{~K}$ (black) and at $2000 \mathrm{~K}$ (red) using the QHA.

from the phonon dispersions of the strained lattices ${ }^{60}$. The main drawback of this method is that since the elastic constants are related to phonon frequencies in the long wavelenght limit, it requires large supercells to converge the sum in Eq. 10. To circumvent this difficulty, we only retain the temperature dependence of Eq.10 that we apply to the static calculations at $0 \mathrm{~K}^{59}$.

We present on Fig. 9, the pressure and temperature evolution of the elastic constants $\left(C_{11}, C_{12}\right.$ and $\left.C_{44}\right)$, the isotropic bulk $\left(K_{T}\right)$ and shear $(G)$ moduli of $\mathrm{MgO}$ for the B1 and B2 phases, calculated using the TDEP method. $K_{T}$ and $G$ were obtained using the Voigt-ReussHill average. For our $0 \mathrm{~K}$ results, we use the stress-strain relations and a deformation matrix applied on the cubic unit cell of the B1 and B2 phases. We also plot on Fig. 9 the results of Karki et al. ${ }^{60,61}$ at $0 \mathrm{~K}$ and at $2000 \mathrm{~K}$ using the QHA (for the $\mathrm{B} 1$ phase). For both phases we observe a softening of $C_{11}$ and $C_{44}$ while $C_{12}$ hardens with temperature. For the $\mathrm{B} 1$ phase, $C_{44}$ shows almost no pressure dependence above $150 \mathrm{GPa}$ and the effect of the temperature is also very weak. In the $\mathrm{B} 2$ phase, $C_{44}$ has a different behavior, it increases with pressure and softens with temperature. Since $K_{T}$ is given by $\left(C_{11}+2 C_{12}\right) / 3$, the effect of temperature on these two elastic constants cancel each other to result in a small decrease of the bulk modulus in temperature for both phases. The shear modulus $G$ evolves in temperature in a similar way than $C_{44}$.

\section{B. Grüneisen parameter}

The Grüneisen parameter $\gamma$ provides a bridge between $P$ and $T$ and is a key quantity for the Earth's interior where $P$ as a function of depth is well constrained, thanks to seismological data, but $T$ is still poorly known. The evolution of $\gamma$ with density, but also with temperature is therefore essential to build reliable EOS for planetary modeling ${ }^{62,63}$. The mode Grüneisen parameters are defined by the volume derivatives of the phonon-mode frequencies :

$$
\gamma_{s}=-\frac{V}{\omega_{s}}\left(\frac{\partial \omega_{s}}{\partial V}\right)_{T}
$$

They can be obtained from the third-order force constants $\Psi_{i j k}$ following ${ }^{40}$ :

$\gamma_{s}(\mathbf{q})=-\frac{1}{6 \omega_{s}^{2}(\mathbf{q})} \sum_{i j k \alpha \beta \gamma} \Psi_{i j k}^{\alpha \beta \gamma} \frac{X_{i s}^{\star \alpha}(\mathbf{q}) X_{j s}^{\beta}(\mathbf{q})}{\sqrt{M_{i} M_{j}}} r_{k}^{\gamma} \exp \left[i \mathbf{q} . \mathbf{R}_{j}\right]$

where $M_{i}$ and $r_{i}^{\alpha}$ are the mass and the $\alpha$ th component of the vector position of atom $i, \omega_{s}$ the frequency of mode $s$, the $X$ 's are the eigenvectors and $\mathbf{R}_{i}$ the lattice vector of the unitcell of atom $i$.

From the $\gamma_{s}$ 's the thermodynamical Grüneisen parameter is obtained by :

$$
\gamma=\frac{\sum_{s=1}^{3 N_{a}} \gamma_{s} C_{V, s}}{C_{V}}
$$

where the $C_{V, s}$ are the mode heat capacities, and $C_{V}$ the specific heat.

In EOS, a commonly used expression for the volume dependence of the Grüneisen parameter has been given by Al'tshuler et al. ${ }^{64}$ :

$$
\gamma=\gamma_{\infty}+\left(\gamma_{0}-\gamma_{\infty}\right) x^{\beta}
$$

where $\gamma_{0}$ is the Grüneisen parameter at ambient conditions, $\gamma_{\infty}$ is the Grüneisen parameter at infinite compression $\left(x=V / V_{0}=0\right)$ and $\beta$ is a fitted parameter. Since $\mathrm{MgO}$ has a long history as an internal pressure standard at extremely high pressure and temperature there is a large collection of parameters for the volume dependance of its Grüneisen parameter. The Mie-Grüneisen model have been used to reproduce experimental $P-V-T$ data and obtain the parameters entering Eq. 14.

We present in Fig. 10 several versions of Eq. 14 based on experimental data ${ }^{8,65-68}$ and the results of A. Oganov et al. ${ }^{69}$ based on theoretical $P-V-T$ points obtained with AIMD, while the corresponding fitting parameters are given in Table I. We also report our values obtained with Eq. 13 for the B1 and B2 phases at 2000 and 8000 $\mathrm{K}$. We have performed an AIMD at $300 \mathrm{~K}$ and at the equilibrium volume of the $\mathrm{B} 1$ phase. We obtain a value 
TABLE I. Fitting parameters of function 14 of the Grüneisen parameter of the $\mathrm{B} 1$ phase of $\mathrm{MgO}$.

\begin{tabular}{|c|c|c|c|}
\hline EOS & $\gamma_{0}$ & $\gamma_{\infty}$ & $\beta$ \\
\hline This work & 1.440 & 0.800 & 3.255 \\
\hline $\mathrm{PAW}^{69}$ & 1.455 & 0.841 & 3.057 \\
\hline Dorogokupets et al. ${ }^{66}$ & 1.50 & 0.75 & 2.96 \\
\hline $\mathrm{Wu}$ et $a .^{8}$ & 1.520 & 0.606 & 1.406 \\
\hline Tange et al. ${ }^{68}$ & 1.431 & 1.016 & 3.50 \\
\hline Speziale et $a l .{ }^{65}$ & 1.524 & 1.325 & 11.8 \\
\hline
\end{tabular}

of 1.44 for the Grüneisen parameter, close to the experimental value of 1.54. Using this value as $\gamma_{0}$ we have fitted our results for the B1 phase at $2000 \mathrm{~K}$ to obtain $\gamma_{\infty}$ and $\beta$, see Table I. Our direct calculations of $\gamma$ are close to the results of $\mathrm{A}$. Oganov et $a l^{69}{ }^{6}$ obtained by fitting the thermal pressure extracted of AIMD simulations performed with GGA. Our results are also in good agreement with the semi empirical EOS proposed by $\mathrm{P}$. I. Dorogokupets et al. ${ }^{66}$. For the B2 phase we find larger values for $\gamma$, about $25 \%$, compared to the B1 phase. We observe a similar difference in the thermal pressure extracted from AIMD simulations. For the B2 phase, the volume dependence of $\gamma$ is rather weak. We performed an AIMD simulation at $9 \mathrm{~g} / \mathrm{cm}^{3}\left(V / V_{0}=0.4\right)$ and we obtain a value of 1.08 .

In the Mie-Grüneisen EOS, the temperature dependence of $\gamma$ at constant $V$ is neglected to directly obtain the thermal pressure in terms of the thermal energy : $P_{\mathrm{TH}}=\gamma E_{\mathrm{TH}} / V$. O. L. Anderson ${ }^{62}$ has shown that this assumption does not hold for $\mathrm{MgO}$. Using experimental data, he obtained, at constant $V$, a value of 1.39 at 1800 $\mathrm{K}$, so $10 \%$ lower than at room temperature. At $8000 \mathrm{~K}$, and for larger compression, we also observed a decrease of $\gamma$ with temperature, see Fig. 10 and 11. The effect is larger for the B1 phase and is almost constant with volume, while for the B2 phase the temperature dependence diminishes with the compression (Fig. 11). This shows the limitation of the Mie-Grüneisen approach to obtain the thermal pressure.

\section{Wave velocities}

From the Grüneisen parameter we can also obtain the volume thermal expansion coefficient defined as:

$$
\alpha_{V}=\frac{\gamma C_{V}}{K_{T} V}
$$

Then, knowing $\gamma$ and $\alpha$, we can obtain the adiabatic incompressibility $K_{S}=(1+\alpha \gamma T) K_{T}$ and with the shear modulus $G$, the compressional and shear wave velocities, $V_{\mathrm{P}}$ and $V_{\mathrm{S}}$ following :

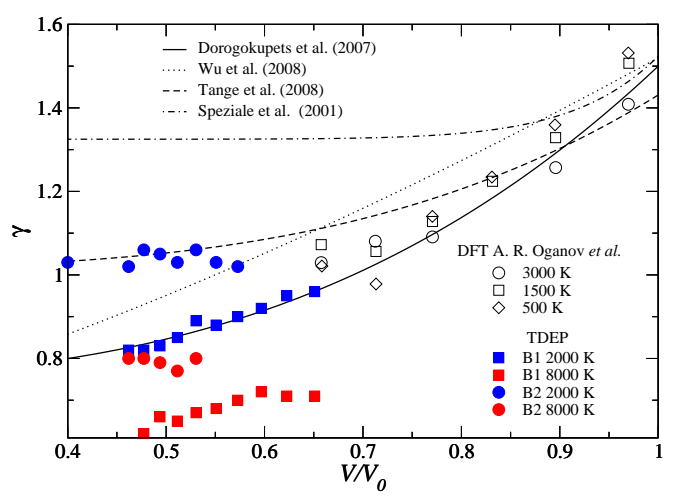

FIG. 10. (Color online) Grüneisen parameter of $\mathrm{MgO}$. The black open symbols are the effective Grüneisen parameter of A. R. Oganov et $a l .{ }^{69}$. The lines are Grüneisen parameters used in semi-empirical equation of state ${ }^{8,65,66,68}$

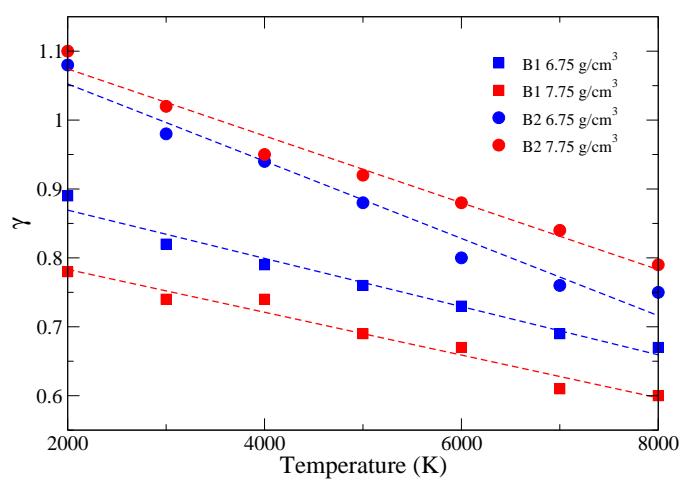

FIG. 11. (Color online) Temperature dependence of the Grüneisen parameter of $\mathrm{MgO}$ for the $\mathrm{B} 1$ (squares) and $\mathrm{B} 2$ (circles) phases at $6.25 \mathrm{~g} / \mathrm{cm}^{3}$ (red) and $7.75 \mathrm{~g} / \mathrm{cm}^{3}$ (blue). The dashed lines are linear fits of these data.

$$
\begin{aligned}
& V_{p}=\sqrt{\frac{K_{S}+4 / 3 G}{\rho}} \\
& V_{s}=\sqrt{\frac{G}{\rho}}
\end{aligned}
$$

Our results are presented in Fig. 12. In both phases, $V_{\mathrm{P}}$ has a similar pressure and temperature dependence while $V_{\mathrm{S}}$ shows a larger pressure and temperature dependence in the B2 phase that are mainly due to the differences observed in the evolution of the elastic constant $C_{44}$ between B1 and B2, see Fig. 9. Compared to iron, temperature has a weaker effects on the wave velocities. B. Martorell et al. ${ }^{70}$ predict a decrease of 8.6 and $29.6 \%$ 


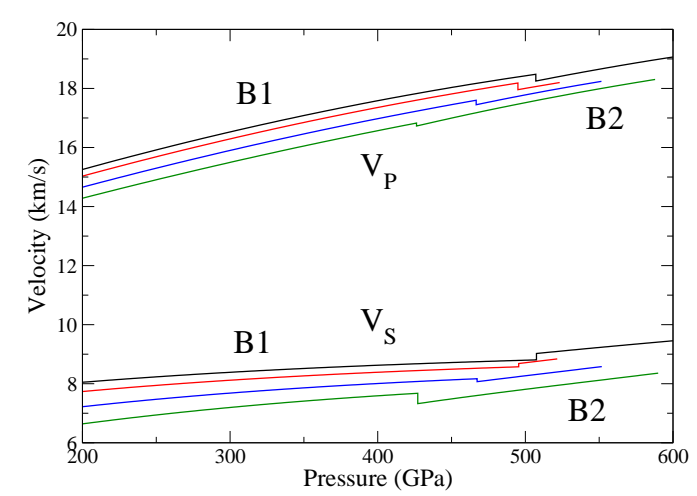

FIG. 12. (Color online) Compressional and shear wave velocities of $\mathrm{MgO}$ as a function of pressure at 0 (black), 2000 (red), 5000 (blue) and $8000 \mathrm{~K}$ (green). The kink in the curves indicates the phase transition between $\mathrm{B} 1$ and $\mathrm{B} 2$.

for $V_{\mathrm{P}}$ and $V_{\mathrm{S}}$ in iron at $6000 \mathrm{~K}$ (before the premelting effect $^{71}$ ) and $360 \mathrm{GPa}$ while we found 4.5 and $9.7 \%$ for the same conditions.

\section{CONCLUSIONS}

Using a recent method to calculate the vibrational contribution to the free energy we have studied the phase transition between the $\mathrm{B} 1$ and $\mathrm{B} 2$ phase in $\mathrm{MgO}$. Compared to previous theoretical works, we obtain a stronger correction to the quasiharmonic approximation at high temperature which pushes the transition line to higher pressures. We show that anharmonic effects cannot be neglected and have to be carefully calculated. We have also estimated the anharmonic part not described by our method and we show that its effect is to further increase the transition pressure. Superimposed on recent shock experiments, our results indicate a direct transition between the $\mathrm{B} 1$ structure towards the liquid along the Hugoniot close to the triple point B1-B2-liquid. New experiments using $\mathrm{x}$-ray diffraction are necessary to confirm this assertion. Using the full capabilities of the TDEP we also derive several thermodynamic quantitites as the elastic constants, the Grüneisen parameters and the sound velocities that are crucial quantities to build reliable high pressure high temperature EOS to describe planetary interiors.

\section{ACKNOWLEDGEMENTS}

We would like to thank X. Gonze for helpful discussions. We also thank M. Desjarlais for sharing with us details of the method used in Ref. 12. This research was supported by the PlanetLab program of the Agence Nationale de la Recherche (ANR) grant ANR-12-BS040015-04.
1 D. Valencia, R. J. O'Connell, and D. Sasselov, Icarus 181, 545 (2006).

2 T. Guillot, Annu. Rev. Earth Planet. Sci. 33, 493 (2005).

${ }^{3}$ H. F. Wilson and B. Militzer, Phys. Rev. Lett 108, 111101 (2012).

4 M. J. Mehl, R. E. Cohen, and H. Krakauer, J. Geophys. Res. 93, 8009 (1988).

5 A. Strachan, T. Çagin, and W. A. Goddard, Phys. Rev. B 60, 15084 (1999).

${ }^{6}$ A. R. Oganov, M. J. Gillan, and G. D. Price, J. Chem. Phys. 118, 10174 (2003).

7 D. Alfè, Phys. Rev. Lett 94, 235701 (2005).

8 Z. Wu, R. M. Wentzcovitch, K. Umemoto, B. Li, K. Hirose, and J.-C. Zheng, J. Geophys. Res. 113, B06204 (2008).

9 A. B. Belonoshko, S. Arapan, R. Martonak, and A. Rosengren, Phys. Rev. B 81, 054110 (2010).

10 B. Boates and S. A. Bonev, Phys. Rev. Lett 110, 135504 (2013).

11 D. Cebulla and R. Redmer, Phys. Rev. B 89, 134107 (2014).

12 S. Root, L. Shulenburger, R. W. Lemke, D. H. Dolan, T. R. Mattsson, and M. P. Desjarlais, Phys. Rev. Lett 115, 198501 (2015).
${ }^{13}$ F. Coppari, R. F. Smith, J. H. Eggert, J. Wang, J. R. Rygg, A. Lazicki, J. A. Hawreliak, G. W. Collins, and T. S. Duffy, Nature geoscience 6, 926 (2013).

14 R. S. McWilliams, D. Spaulding, J. H. Eggert, P. Celliers, D. G. Hicks, R. F. Smith, G. W. Collins, and R. Jeanloz, Science 338, 1330 (2012).

15 R. M. Bolis, G. Morard, T. Vinci, A. Ravasio, E. Bambrink, M. Guarguaglini, M. Koenig, R. Musella, F. Remus, J. Bouchet, N. Ozaki, K. Miyanashi, T. Sekine, Y. Sakawa, T. Sano, R. Kodama, F. Guyot, and A. Benuzzi-Mounaix, Geophys. Res. Lett. 43, 9475 (2016).

16 K. Miyanishi, Y. Tange, N. Ozaki, T. Kimura, T. Sano, Y. Sakawa, T. Tsuchiya, and R. Kodama, Phys. Rev. E 92, 023103 (2015).

17 D. M. Ceperley and B. J. Alder, Phys. Rev. Lett. 45, 566 (1980).

18 J. P. Perdew, K. Burke, and M. Ernzerhof, Phys. Rev. Lett 77, 3865 (1996).

19 R. Armiento and A. E. Mattsson, Phys. Rev. B 72, 085108 (2005).

20 X. Gonze, Phys. Rev. B 55, 10337 (1997).

21 X. Gonze and C. Lee, Phys. Rev. B 55, 10355 (1997). 
22 S. Baroni, S. de Gironcoli, , A. D. Corso, and P. Gianozzi, Rev. Mod. Phys. 73, 515 (2001).

${ }^{23}$ K. Parlinski, J. Łażewski, and Y. Kawazoe, J. Phys. Chem. Solids 61, 87 (2000).

24 B. Fultz, Prog. Mater. Sci. 55, 247 (2010).

25 P. Souvatzis, O. Eriksson, M. I. Katsnelson, and S. P. Rudin, Phys. Rev. Lett 100, 095901 (2008).

26 O. Hellman, I. A. Abrikosov, and S. I. Simak, Phys. Rev. B 84, 180301 (2011).

27 J. Bouchet and F. Bottin, Phys. Rev. B 95, 054113 (2017).

28 B. Dorado, F. Bottin, and J. Bouchet, Phys. Rev. B 95, 104303 (2017).

29 A. Glensk, B. Grabowski, T. Hickel, and J. Neugebauer, Phys. Rev. Lett 114, 195901 (2015).

${ }^{30}$ B. Grabowski, L. Ismer, T. Hickel, and J. Neugebauer, Phys. Rev. B 79, 134106 (2009).

31 A. Erba, M. Shahrokhi, R. Moradian, and R. Dovesi, J. Chem. Phys. 142, 044114 (2015).

32 Z. Wu and R. M. Wentzcovitch, Phys. Rev. B 79, 104304 (2009).

33 I. Errea, M. Calandra, and F. Mauri, Phys. Rev. Lett 111, 177002 (2013).

${ }^{34}$ I. Errea, M. Calandra, and F. Mauri, Phys. Rev. B 89, 064302 (2014).

35 O. Hellman, P. Steneteg, I. A. Abrikosov, and S. I. Simak, Phys. Rev. B 87, 104111 (2013).

36 J. Bouchet and F. Bottin, Phys. Rev. B 92, 174108 (2015).

37 T. Tadano, Y. Gohda, and S. Tsuneyuki, J. Phys.: Condens. Matter 26, 2254012 (2014).

${ }^{38}$ L. J. Nelson, G. L. W. Hart, F. Zhou, and V. Ozolinš, Phys. Rev. B 87, 035125 (2013).

39 K. Esfarjani and H. T. Stokes, Phys. Rev. B 77, 144112 (2008).

40 O. Hellman and I. A. Abrikosov, Phys. Rev. B 88, 144301 (2013)

41 The ABINIT code is a common project of the Catholic University of Louvain (Belgium), Corning Incorporated, CEA (France) and other collaborators (URL http://www.abinit.org).

42 X. Gonze, B. Amadon, P.-M. Anglade, J.-M. Beuken, F. Bottin, P. Boulanger, F. Bruneval, D. Caliste, R. Caracas, M. Côté, T. Deutsch, L. Genovese, P. Ghosez, M. Giantomassi, S. Goedecker, D. Hamann, P. Hermet, F. Jollet, G. Jomard, S. Leroux, M. Mancini, S. Mazevet, M. Oliveira, G. Onida, Y. Pouillon, T. Rangel, G.-M. Rignanese, D. Sangalli, R. Shaltaf, M. Torrent, M. Verstraete, G. Zerah, and J. Zwanziger, Computer Physics Communications 180, 2582 (2009).

43 D. J. Hooton, Z. Phys. 142, 42 (1955).

44 A. Dewaele, V. Stutzmann, J. Bouchet, F. Bottin, F. Occelli, and M. Mezouar, Phys. Rev. B 91, 134108 (2015).

45 P. E. Blöchl, Phys. Rev. B 50, 17953 (1994).

46 M. Torrent, F. Jollet, F. Bottin, G. Zerah, and X. Gonze, Comput. Mater. Sci. 42, 337 (2008).
47 N. Holzwarth, A. Tackett, and G. Matthews, Comput. Phys. Commun. 135, 329 (2001).

48 ATOMPAW is a general license public code developed at Wake Forest University. Some of its capabilities have been developed at the Commissariat à l'énergie atomique (http://pwpaw.wfu.edu).

49 A. Dewaele, M. Torrent, P. Loubeyre, and M. Mezouar, Phys. Rev. B 78, 104102 (2008).

50 M. Millot, N. Dubrovinskaia, S. B. A. Černok, L. Dubrovinsky, D. G. Braun, P. M. Celliers, G. W. Collins, J. H. Eggert, and R. Jeanloz, Science 347, 418 (2015).

${ }^{51}$ D. E. Fratanduono, M. Millot, R. G. Kraus, D. K. Spaulding, G. W. Collins, P. M. Celliers, and J. H. Eggert, Phys. Rev. B 97, 214105 (2018).

52 T. Taniuchi and T. Tsuchiya, J. Phys.: Condens. Matter 30, 114003 (2018).

53 N. de Koker and L. Stixrude, Geophys. J. Int. 178, 162 (2009).

54 A. B. Belonoshko, S. Davis, N. V. Skorodumova, P. H. Lundow, A. Rosengren, and B. Johansson, Phys. Rev. B 76, 064121 (2007).

55 R. Musella, S. Mazevet, and F. Guyot, Phys. Rev. B 99, 064110 (2019).

56 J. Bouchet, F. Bottin, G. Jomard, and G. Zérah, Phys. Rev. B 80, 094102 (2009).

57 G. Liebfried and W. Ludwig, Solid State Physics, edited by N. Y. Academic Press (1961).

58 D. C. Wallace, Thermodynamics of Crystals, edited by P. Ziesche and H. Eschrig (Willey, New York, 1972).

59 N. Shulumba, O. Hellman, L. Rogström, Z. Raza, F. Tasnádi, I. A. Abrikosov, and M. Odén, Appl. Phys. Lett. 107, 231901 (2015).

60 B. B. Karki, R. M. Wentzcovitch, S. de Gironcoli, and S. Baroni, Science 286, 1705 (1999).

61 B. B. Karki, Phys. Earth Planet. Inter. 240, 43 (2015).

62 O. L. Anderson, Geophys. J. Int. 143, 279 (2000).

${ }^{63}$ F. D. Stacey, Rep. Prog. Phys. 68, 341 (2005).

${ }^{64}$ L. V. Al'tshuler, S. E. Brusnikin, and E. A. Kuz'menkov, J. Appl. Mech. Tech. Phys. 28, 129 (1987).

65 S. Speziale, C. Zha, T. S. Duffy, R. J. Hemley, and H. K. Mao, J. Geophys. Res. 106, 515 (2001).

66 P. I. Dorogokupets and A. Dewaele, High Pressure Res. 27, 431 (2007).

67 P. I. Dorogokupets, Phys. Chem. Minerals 37, 677 (2010).

68 Y. Tange, Y. Nishihara, and T. Tsuchiya, J. Geophys. Res. 114, B03208 (2009).

69 A. R. Oganov and P. I. Dorogokupets, Phys. Rev. B 67, 224110 (2003).

70 B. Martorell, I. G. Wood, J. Brodholt, and L. Vočadlo, Earth Planet. Sci. Lett. 451, 89 (2016).

71 B. Martorell, L. Vočadlo, J. Brodholt, and I. G. Wood, Science 342, 466 (2013). 